\title{
Changes in Quality of Life and Working Capacity Before and After Kidney Transplantation
}

\author{
F.J. Shih, P.H. Lee, J.D. Wang, R.H. Hu, M.K. Lai, H.Y. Lin, M.H. Lin, and C.J. Lee
}

KIDNEY transplantation (KT) has evolved as a successful therapy for patients struggling with end-stage renal disease. Advances in surgical and medical protocols have resulted in the excellent survival rates. ${ }^{1,2}$ Debates regarding the quality of life (QOL) of dialysis and transplant recipient groups have been ongoing for about two decades. ${ }^{3-11}$ Although most studies show that QOL is one of the most important benefits of KT, ${ }^{3,7-24}$ some researchers still argue that KT does not significantly improve QOL over dialysis. $^{4-6}$ The health-related QOL of KT recipients is a complex phenomenon. Recipients' assessment of QOL often reflects their adaptation to their postdischarge, posttransplant lives. During the predischarge stage, most Taiwanese KT recipients are anxious about the quality of medical care, their self-efficacy, and related social support. Alleviating the anxiety of KT recipients with regard to these issues may be critical in determining their recovery and QOL after discharge. ${ }^{25}$ However, few researchers have prospectively monitored the monthly changes in the selfrated QOL of KT recipients during the first postdischarge year. Instead, the QOL is often examined from a crosssectional view, according to the time frame of standard posttransplant evaluation visits; these evaluations tend to center around the medium- or long-term survivors. ${ }^{4-6,8-18}$

Working capacity (WC) as well as income are important indicators of self-assessed QOL among Taiwanese KT recipients. ${ }^{2}$ However, the challenge of transplantation to a recipient's postoperative economic status, and the relationship between recipients' QOL and WC during the first posttransplant year have not been carefully investigated. The purpose of this study was to examine the trend of changes in the QOL and WC after KT, from the preoperative stage to 1 year after discharge from the hospital after KT.

\section{PATIENTS AND METHODOLOGY}

A descriptive, prospective design was used to explore the trends in QOL and WC perceived by KT recipients before and after surgery. The inclusion criteria were as follows: (1) at least 18 years old; (2) clear consciousness; (3) no transplant surgeries other than KT; and (4) prepared to be discharged from National Taiwan University Hospital the next day.

The first interview was conducted 1 day before the patient's discharge from the hospital, and follow-up interviews were conducted 1, 2, 3, 6, and 12 months after discharge from the hospital during outpatient, posttransplant visits. The patient profile was used to collect information about the patient's demographic information, medical history, medications that the subjects had taken in the floor unit, postoperative complications, and the length of floor unit stay. The subjects were asked to rate their perceptions of QOL and WC on separate visual analog scales (VASs). The VAS used in this study was a 100 -millimeter $(\mathrm{mm})$ vertical line. This line was anchored at the bottom with the words "Worst quality of life" or "Unable to work," and at the top by "Optimal quality of life" or "Optimal working capacity," respectively. During the first interview, the participants were also asked to rate their perceptions of overall preoperative QOL and WC on VASs, based on their 24 hours preoperatively.

\section{RESULTS}

Patient Demographic Data

The numbers of patients who participated in the six interviews were 50, 48, 46, 44, 40, and 33, respectively. During the first interview, the participants' ages ranged from 19 to 59 (mean 38.10) years. Sixty-two percent of the patients were married, $28 \%$ were single, and $10 \%$ were divorced or widowed. Seventy percent considered themselves were religious (66\% Buddhist, $4 \%$ Protestant). Fifty-two percent of the subjects were employed preoperatively; $6 \%$ worked 4 days, $10 \%$ worked 3 days, $20 \%$ worked 2 days, and $16 \%$ worked 1 day per week. Of the $48 \%$ of the subjects who were unemployed preoperatively, $14 \%$ of whom were housewives, $20 \%$ reported that they were able to work but chose not to; $16 \%$ were unable to work but capable of self-care; and $12 \%$ had irregular working days. Finally, 50\% of the subjects reported having irregular family incomes, and $43 \%$ of the subjects' monthly family income was between US\$469 and US\$1,875 (NT\$32= US\$1) preoperatively.

From the College of Medicine, School of Nursing (F.J.S., H.Y.L., M.H.L.), Department of Surgery (P.H.L., R.H.H., C.J.L.), College of Public Health (J.D.W.), and the Department of Urology (M.K.L.), National Taiwan University, Taipei, Taiwan.

Address reprint requests to Chun-Jean Lee, MD, Professor, National Taiwan University, College of Medicine, Department of Surgery, No. 1, Section 1, Jen-Ai Road, Taipei 10018, Taiwan. E-mail: fujin@ha.mc.ntu.edu.tw. 
Table 1. Comparison Between Quality of Life and Working Capacity by Different Stage Groups

\begin{tabular}{|c|c|c|c|c|c|}
\hline \multirow[b]{2}{*}{ Stage } & \multirow[b]{2}{*}{$\mathrm{N}$} & \multirow{2}{*}{$\frac{\mathrm{QOL}(\%)}{\text { Mean } \pm \mathrm{SD}}$} & \multirow{2}{*}{$\frac{W C(\%)}{\text { Mean } \pm S D}$} & \multirow[b]{2}{*}{$t$ value } & \multirow[b]{2}{*}{ F value } \\
\hline & & & & & \\
\hline (A) Preop day & 50 & $54 \pm 18$ & $64 \pm 19$ & 0.32 & \\
\hline (B) Postop, 1 day before discharge & 50 & $67 \pm 26$ & $65 \pm 21$ & 0.96 & 2.96 \\
\hline (C) Postop, 1 month after discharge & 48 & $77 \pm 18$ & $71 \pm 22$ & 0.82 & 6.33 \\
\hline (D) Postop, 2 months after discharge & 46 & $81 \pm 12$ & $75 \pm 16$ & 0.95 & 8.83 \\
\hline (E) Postop, 3 months after discharge & 44 & $82 \pm 16$ & $78 \pm 14$ & 0.72 & 9.47 \\
\hline (G) Postop, 12 months after discharge & 33 & $83 \pm 18$ & $80 \pm 18$ & 0.39 & 8.99 \\
\hline
\end{tabular}

\section{Changes in the Perceived Health Related QOL}

The mean scores of the perceived health related QOL were $54 \%$ preoperatively, $67 \%$ predischarge, $77 \%$ at 1 month, $81 \%$ at 2 months, $82 \%$ at 3 months, $83 \%$ at 6 months, and $83 \%$ at 12 months after discharge (Table 1). Changes in the perceived QOL between the preoperative stage and each of the six postoperative recovery stages were examined. Major changes in the perceived QOL were noted only between the preoperative stage and 1 month postdischarge $(t=-4.35$, $P<.01)$, and the preoperative stage and 12 months postdischarge $(t=-4.43, P<.01)$. Nevertheless, no significant differences in the changes in the perceived QOL scores across the seven time points were identified with one-way ANOVA $(F=14.71, \mathrm{df}=6274, P>.05)$.

\section{Changes in Perceived WC}

The mean WC scores were $64 \%$ preoperatively, $65 \%$ predischarge, $71 \%$ at 1 month, $75 \%$ at 2 months, $78 \%$ at 3 months, $80 \%$ at 6 months, and $80 \%$ at 12 months after discharge (see Table 1). Differences in the perceived WC were significant between the preoperative and each of the postdischarge stages: 1 month $(t=-2.99), 2$ months $(t=$ $-3.00), 3$ months $(t=-3.01), 6$ months $(t=-2.67)$, and 12 months $(t=-3.87)$. The trend in the improvement of the perceived WC was significant from the preoperative stage to the 3-month postdischarge stage $(\mathrm{F}=4.57, P<.01)$. In addition, the mean perceived WC score was slightly lower than the mean score of perceived QOL across the six postoperative stages, although no correlation between the QOL and WC was identified (Table 1).

The percentages of the subjects employed during the study period were as follows: $52 \%$ preoperatively, $52 \%$ predischarge, $48 \%$ at 1 month, $50 \%$ at 2 months, $54 \%$ at 3 months, $60 \%$ at 6 months, and $67 \%$ at 12 months after discharge. The percentage of the subjects with monthly family incomes of more than US\$469 were 52\% preoperatively and predischarge, and $46 \%$ at 1 month, $50 \%$ at 2 months, $52 \%$ at 3 months, $65 \%$ at 6 months, and $67 \%$ at 12 months after discharge (Fig. 1). The trend of changes in the reported employment and economic status across the seven time points indicated that the first month after discharge from the hospital was the stage of lowest employment and family income. Thereafter, the percentage of the subjects employed and their family incomes steadily increased in the subsequent recovery stages, through the twelfth month after discharge from the hospital.

\section{DISCUSSION}

Socioeconomic status as well as race and gender influence how transplant recipients view themselves. ${ }^{24-26}$ Neverthe-
Fig 1. Changes in QOL, WC, employment, and monthly family incomes before and after kidney transplantation.

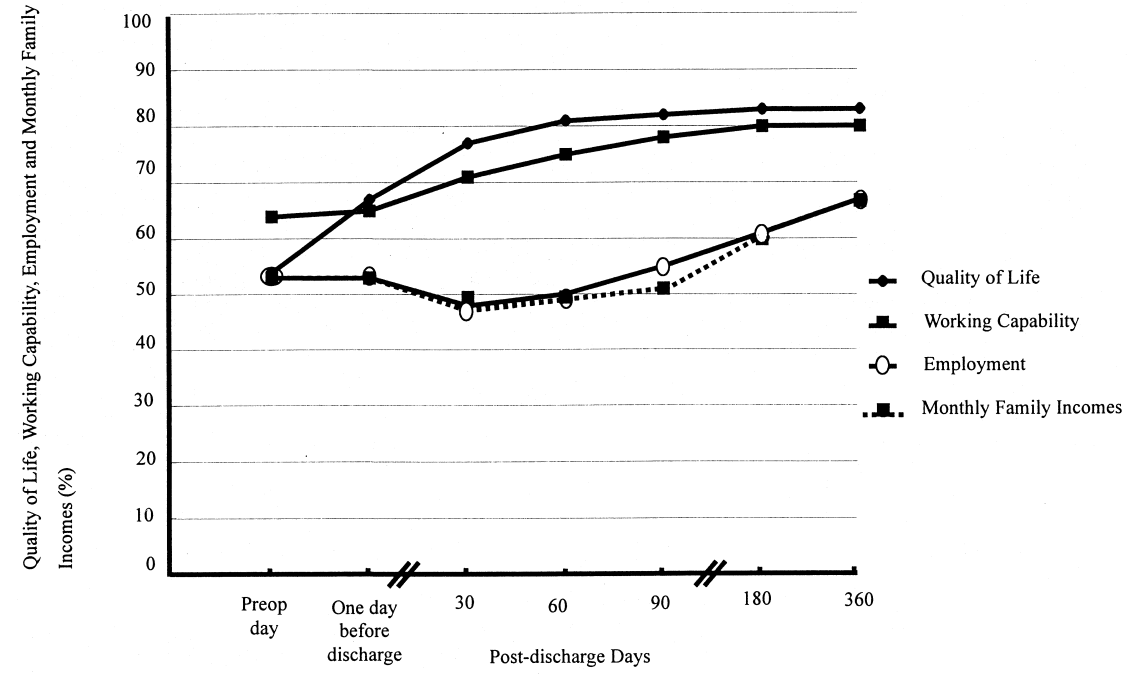


less, the relationship between the changes in the recipients' socioeconomic status and QOL during the first posttransplant year is rarely considered by investigators. Because the correlation of patient and physician assessments of QOL after $\mathrm{KT}$ is low, ${ }^{14}$ we used a VAS scale to allow KT recipients to give subjective appraisals of their global QOL and WC.

Park et $\mathrm{al}^{7}$ used a set of questionnaires to compare the changes in the self-assessed QOL 1 to 10 days preoperatively and 3 months postoperatively. The recipients' emotional and physical QOL scores were found to be significantly better 3 months postoperatively. In our study, no significant differences in the QOL scores were identified when we compared the QOL preoperatively and at 3 months after discharge from the hospital. Rather, the QOL scores were significantly better in the first month (77\%) and at 12 months $(83 \%)$ after discharge compared to the preoperative values (54\%). QOL scores were also found to improve consistently from the preoperative stage through the six postoperative stages, although no correlation between QOL and time was identified in the first posttransplant year.

Complex multidimensional aspects underline concept of global perceptions of QOL examined in this study. ${ }^{1-24}$ QOL is often related to patients' social roles as well as their support systems in both the family and community. Most Taiwanese recipients would not be discharged from the hospital until they had demonstrated themselves to be well prepared for it. From empirical observation, most subjects in this study experiences less physical discomfort and had improved physical competency at the end of the first month after discharge from the hospital. This may be the reason most of the subjects reported higher QOL scores after discharge compared with their QOL scores in the preoperative stage, at which time they had assumed a sick role for a long period.

The subjects in this study also reported significantly higher QOL scores at the end of the first year after discharge compared with their QOL scores in the preoperative stage. This may be because these subjects had good graft function and better social rehabilitation. ${ }^{14,16}$ Good graft function is the result of successful posttransplant medical and nursing protocols (followed monthly in our study), high subject compliance with protocols, and adequate social support. Social rehabilitation can be a result of improved physical competency. In our study, about $70 \%$ of the subjects had resumed their family and other social roles either partially or completely by the end of the twelfth month after discharge from the hospital, roles in which they had been limited prior to surgery. In addition, improvements in QOL may also be attributed in part to the determination of patients to take advantage of the opportunity provided by the transplantation, despite any hardships they may have to endure. ${ }^{27}$

Employment was cited as one of the factors influencing QOL among Taiwanese KT recipients. ${ }^{27}$ Meanwhile, monthly family income has also been reported to influence
Taiwanese recipients' perceptions of their recovery from $\mathrm{KT}^{25}$ From the trend of changes in employment and economic status across the seven stages, the first month after discharge from the hospital appeared to be a critical stage during which patients reported the lowest WC, employment rate, and family income. After this stage, the percentage of the subjects employed, their family incomes, and their perceived WC steadily increased in the subsequent months throughout the end of the study period. The employment rate did not begin to increase until the third month after discharge from the hospital. The need for this delay may be related to the fact that the subjects placed a higher priority on recovery from transplantation than on their economic needs. ${ }^{15}$ The availability of financial aid from National Health Insurance, which was instituted in 1995 and covers most of the medical expenses for KT recipients, may have also contributed to the delay by reducing the financial pressure on patients to return to work.

In conclusion, Taiwanese KT recipients experienced better QOL and WC during the first posttransplant year than preoperatively. Significant changes in QOL were noted at 1 and 12 months after discharge from the hospital, and significant changes in perceived WC were reported between the preoperative stage and each of the postdischarge stages. Researchers are suggested to further explore (a) factors that might contribute to or threaten patients' perceptions of QOL in the early posttransplantation stages; (b) the impact of WC, degree of recovery, and financial status on recipients' QOL; (c) the optimal time for recipients to re-enter the community; and (d) what groups are most vulnerable to poor perceived QOL and WC during their first posttransplant year, and why.

\section{ACKNOWLEDGMENT}

The investigators' thanks go to the women and men who participated in this study. Financial support was graciously provided by the National Science Council, Republic of China (NSC86-2314-B002-294).

\section{REFERENCES}

1. Lee HM: Transplant Proc 28:1171, 1996

2. Hu RH, Lee PH, Tsai MK, et al: Transplant Proc 30:3617, 1998

3. Adang EMM, Kootstra G, Engel GL, et al: Transplant Int 11:11, 1998

4. Sayang R, Kaplan De-Nour A, Shapira Z, et al: Nephron 54:214, 1990

5. Chowanec GD, Binik YM: Soc Sci Med 28:971, 1989

6. Kaplan De-Nour A, Shanan J: Nephron 25:117, 1980

7. Park IH, Yoo HJ, Han DJ, et al: Transplant Proc 28:1937, 1996

8. Evans RW, Manninen DL, Garrison LP, et al: N Engl Med 312:553, 1985

9. Hart LG, Evans RW: J Chron Dis 40(suppl):117s, 1987

10. Slama H, Fisch HU, Frey FJ: Schweiz Rundsch Med Prax 82:1253, 1993 
11. Simmons RG, Anderson C, Kamstra L: Am J Kidney Dis 4:253, 1984

12. Gerber R: Schweiz Med Wschr 119:1113, 1989

13. Thomson NM, Scott DF, Cesnik B, et al: Transplant Proc 21:2184, 1989

14. Witzke O, Becker G, Franke G, et al: Transplant Proc 29:1569, 1997

15. Lin ZC, Liu SY, Liou Chang GL: Nur Res NAROC 1:369, 1993

16. Pisani F, Vennarecci G, Tisone G, et al: Transplant Proc 29:2812, 1997

17. Simmons RG, Abress L, Anderson CR: Transplantation 45:415, 1988

18. Gorlen T, Ekberg O, Abdelnoor M, et al: Scand J Nephrol 27:89, 1993

19. Hathaway D, Strong M, Ganza M: Am Nephrol Nurse Assoc J $17: 433,1990$
20. Hathaway D, Hartwig M, Winsett RP, et al: Am Nephrol Nurse Assoc J 19:152, 1992

21. Huang SH, Hong JJ, Lan CF, et al: Chinese Renal Med J $5: 234,1991$

22. Lin CS, Liou SE, Liou-Chang GL: NAROC Nurs Res 1:369, 1993

23. White MJ, Ketefian S, Starr AJ, et al: Am Nephrol Nurse Assoc J 17:421, 1990

24. Johnson CD, Wicks MN, Misltead J, et al: Image J Nurse Schol 30:125, 1998

25. Shih FJ, Lin MH, Lin HY, et al: Transplant Proc 30:3639, 1998

26. Simmons RG, Marine SK, Simmons RL: Gift of life: The effect of organ transplantation on individual, family, and societal dynamics. New Brunswick, NJ: Transaction, Inc. 1987

27. Huang SH: Taipei, Taiwan: Yang-Ming University, College of Public Health, Master Thesis (in Chinese) 1991 